\title{
Immunohistochemical assessment of protein phosphorylation state: the dream and the reality
}

\author{
James W. Mandell
}

Accepted: 30 June 2008 / Published online: 22 July 2008

(C) Springer-Verlag 2008

\begin{abstract}
The development of phosphorylation state-specific antibodies (PSSAs) in the 1980s, and their subsequent proliferation promised to enable in situ analysis of the activation states of complex intracellular signaling networks. The extent to which this promise has been fulfilled is the topic of this review. I review some applications of PSSAs primarily in the assessment of solid tumor signaling pathway activation status. PSSAs have received considerable attention for their potential to reveal cell type-specific activation status, provide added prognostic information, aid in the prediction of response to therapy, and most recently, demonstrate the efficacy of kinase-targeted chemotherapies. However, despite some successes, many studies have failed to demonstrate added value of PSSAs over general antibody immunohistochemistry. Moreover, there is still a large degree of uncertainty about the interpretation of complex and heterogeneous staining patterns in tissue samples and their relationship to the actual phosphorylation states in vivo. The next phase of translational research in applications of PSSAs will entail the hard work of antibody validation, gathering of detailed information about epitope-specific lability, and implementation of methods for standardization.
\end{abstract}

Keywords Phosphospecific · Protein phosphorylation · Immunohistochemistry $\cdot$ Kinases

J. W. Mandell ( $\square)$

Department of Pathology,

University of Virginia School of Medicine,

P.O. Box 800904, Charlottesville, VA 22908, USA

e-mail: jwm2m@virginia.edu

\section{Introduction}

Reversible protein phosphorylation was discovered over 50 years ago by Fischer and Krebs, during their studies on the enzyme glycogen phosphorylase (Fischer and Krebs 1955). Phosphorylation and its reverse reaction, dephosphorylation, are carried out by over 500 kinases (Manning et al. 2002) and a smaller number of phosphatases, respectively. An explosion of work on kinases, phosphatases and their numerous protein substrates has generated an immense body of biochemical knowledge; a current PUBMED search using the search term "phosphorylation" finds over 150,000 articles. Until the development of phosphorylation state-specific antibodies (PSSAs), however, the study of protein phosphorylation required radioactive phosphate incorporation assays and was largely limited to biochemistry labs. PSSAs made possible the study of protein phosphorylation in situ, allowing cell biologists, histologists and pathologists at least a static view of dynamic protein phosphorylation reactions in the spatially complex structures of cells and tissues. In this review I focus on recent advances in applications of PSSAs, focusing on studies of solid tumors that include immunohistochemical approaches. I will then address some technical and practical limitations in the methodology, and suggest future directions to improve the data arising from use of these reagents.

Historical aspects of the development of PSSAs were discussed in a previous review (Mandell 2003). One key reference omission in that article, however, was the first report of a PSSA, which recognized the phosphorylated form of a substrate of cGMP-dependent protein kinase (Nairn et al. 1982). This was one piece of a large body of work by Paul Greengard's group on protein phosphorylation in the nervous system, recognized with the awarding of a Nobel prize in 2000 (De Camilli and Carew 2000). 
The number of commercially available PSSAs has steadily grown, as has the number of publications utilizing these reagents in immunohistochemical applications (Fig. 1). Despite this growth, there are still many unanswered questions about the robustness and reproducibility of immunohistochemical assays of protein phosphorylation, which will be discussed. The focus will be on immunohistochemical studies of human solid tumors over the past 5 years. Studies reviewed include novel morphological observations on cellular signaling pathway activation, as well as the potential for PSSAs to provide prognostic information, prediction of response to targeted therapies, and post-treatment assessment of response to therapy. Due to space limitations only a sampling of the large body of work is discussed here.

\section{The dream}

In the realm of solid tumor assessment, the dream offered by PSSAs is the ability to provide robust multiparameter measurements of intracellular signaling pathway status, in a way that provides prognostic, predictive, and/or therapeutic information to the clinician. An example of a multiparameter panel of PSSAs applied to a human glioblastoma is shown in Fig. 2 (unpublished data). In this case, overexpression of total EGFR was present (compared to no signal in adjacent nonneoplastic brain). In addition, strong tyrosine phosphorylation of the receptor was found at Tyr1173 but only focal weak phosphorylation at Tyr1068. Downstream kinases ERK and mTOR show strong cytoplasmic

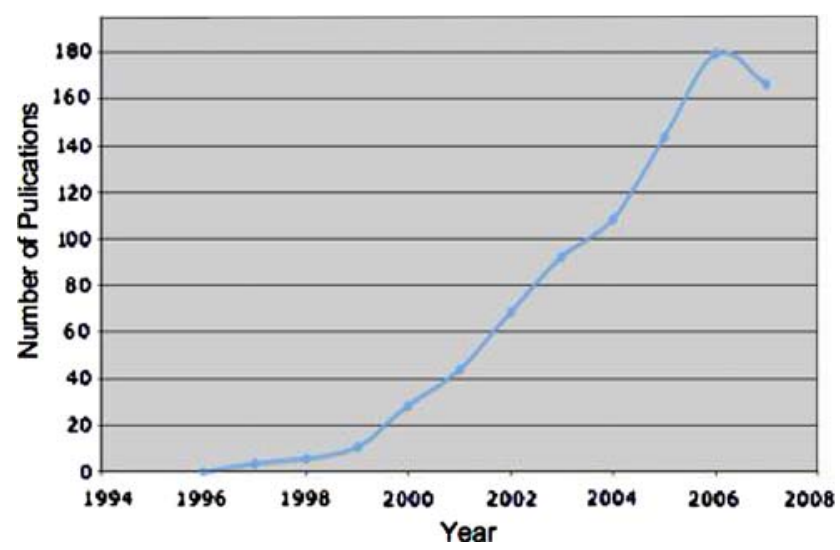

Fig. 1 Continuous growth of publications with immunohistochemical applications of PSSAs. A Google Scholar Advanced search (full text search) using the Boolean search: $\{$ ("phosphospecific" OR "phosphospecific" OR "phosphorylation-specific" OR "phosphorylation statespecific") AND "immunohistochemistry"\} revealed a continuous increase in the annual number of publications including these key phrases in the full text content. The apparent slight decline in 2007 is likely due to the restricted full text access of many journals in the first year after publication phosphorylation in a subset of tumor cells, whereas the transcription factor CREB is strongly phosphorylated in the majority of tumor cells. Phospho-Histone (H3) is an indicator of mitotic activity. Phosphorylation of the actin-associated proteins Ezrin/Radixin/Moesin, potential mediators of tumor invasiveness, is strong in all cells. Finally, a generic anti-phospho-tyrosine antibody is used to assess the general preservation of tyrosine phosphorylation in this tumor sample. In the dream, we can provide quantitative measures of protein phosphorylation, both in terms of percent of protein phosphorylated, as well as in terms of percent cells positive (above a stated threshold). Additionally, the analysis will include information about subcellular localization of the phosphoepitopes under study. All this information is then entered into an algorithm that classifies the tumor among others of its general type, providing the oncologist with robust and clinically useful information about the activation profile of signaling networks in this patient's tumor. But now we must awaken.

\section{The reality}

PSSAs as prognostic markers

A central question in the field is whether knowledge about the phosphorylation (activation) state of a protein, in addition to its general level of expression, provides nonredundant information. Several studies on human solid tumors, most focusing on EGFR family members, have begun to address this question. Because immunohistochemistry for total EGFR failed to demonstrate prognostic importance in non-small cell lung carcinoma (NSCLC), it was hypothesized that information about the activation (phosphorylation) of EGFR could reveal prognostic significance. To address this, immunohistochemistry for total and phosphoEGFR as well as phospho-STAT3 was performed on archival NSCLC cases. Of the EGFR-expressing tumors, approximately $1 / 3$ showed pEGFR immunoreactivity, but the presence of pEGFR did not provide statistically significant prognostic information (Cortas et al. 2007).

In a similar study on locally advanced breast cancer, total EGFR expression correlated with negative hormone receptor status, and was associated with significantly worse relapse-free survival. However, the authors found no association of phospho-EGFR with outcome (Nieto et al. 2007).

A large body of evidence indicates that mutated BRAF and NRAS contribute to the genesis and growth of melanomas, presumably by activation of the extracellular signalregulated kinase (ERK) pathway. The presence of phosphorylated ERK1/2 was analyzed in 170 melanomas with established NRAS/BRAF mutational status. The authors found notable heterogeneity of phospho-ERK staining with 


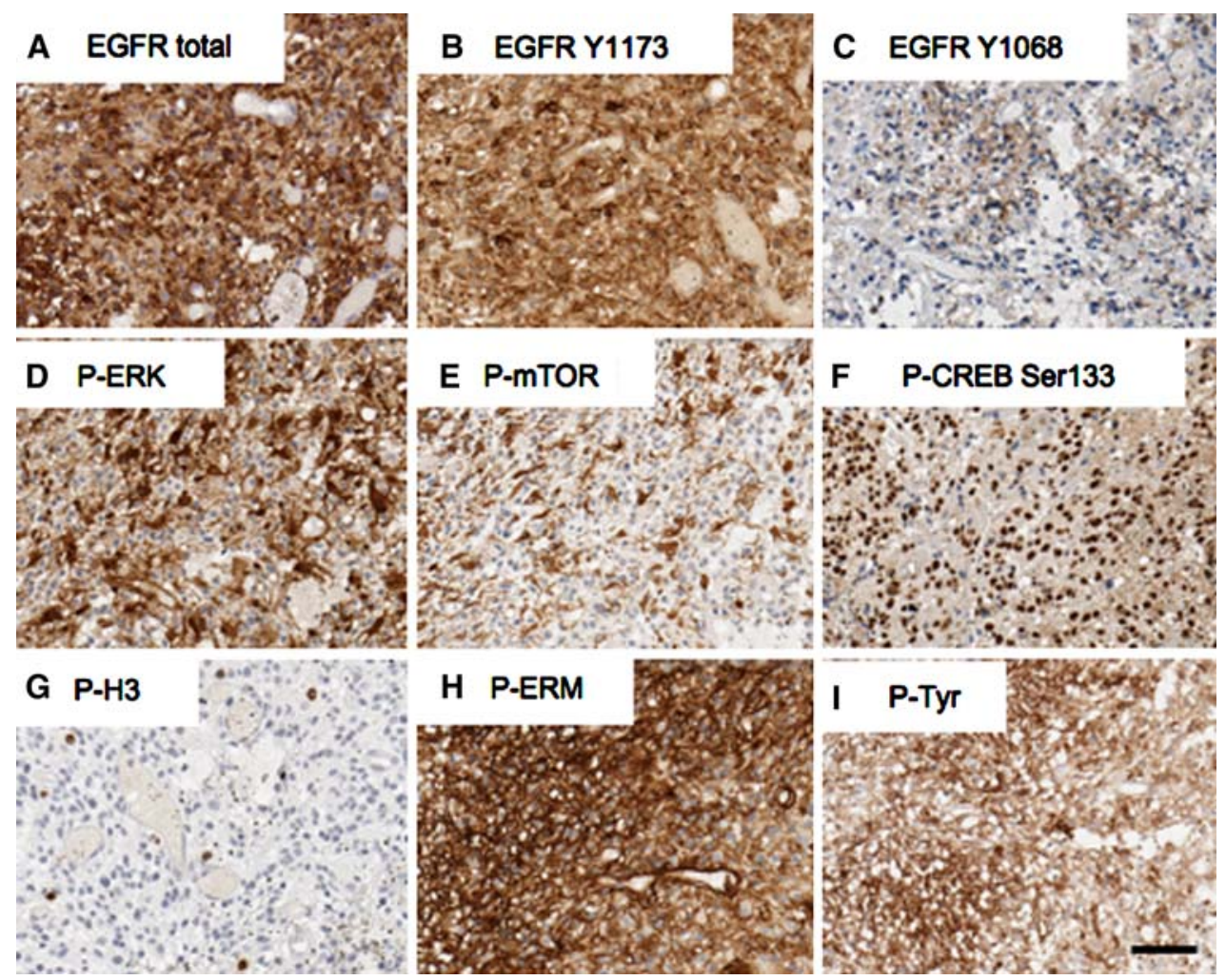

Fig. 2 An example of a panel of phosphorylation state-specific antibodies applied to a human glioblastoma multiforme. a Overexpression of total EGFR is apparent [(compared to little or no signal in adjacent nonneoplastic brain, (not shown)]. In addition, strong tyrosine phosphorylation of the receptor was found at Tyr1173 (b) but only focal weak phosphorylation at Tyr1068 (c). Downstream intracellular kinases ERK (d) and mTOR (e) show strong cytoplasmic and phosphoryla-

only a minority of tumor cells positive in most cases. Moreover, the percentage of phospho-ERK-positive tumor cells did not correlate with prognosis (Houben et al. 2008).

A frequently overlooked phenomenon is staining of nontumor stromal cells and the vasculature with PSSAs. A large body of evidence points at the tumor microenvironment as critical to oncogenesis (Roskelley and Bissell 2002). In addition, vascular-targeted therapies are now in common use, making PSSA assessment of stromal cell and vascular cell signaling a possibly ripe area of investigation. Mouse xenograft studies were performed with tumors that produced TGF-alpha, stimulating endothelial cells to express and activate EGFR (Kuwai et al. 2008). EGFR blockade in these tumors lead to endothelial cell apoptosis and subsequent tumor necrosis. Importantly, tumor xenografts in which TGF-alpha was knocked down with shRNA did not stimulate endothelial EGFR activation, and these tumors were resistant to therapy. Based on this and other similar studies, the assessment of human tumor endothelial cell EGFR, PDGFR or VEGFR activation status could provide clinically valuable information, but to date has not been specifically analyzed. tion in a subset of tumor cells, whereas the transcription factor CREB (f) is strongly phosphorylated in the nuclei of most tumor cells. Phospho-Histone $\mathrm{H} 3$ (g) is a marker of all cells in mitosis. Phosphorylation of the actin-associated proteins Ezrin/Radixin/Moesin, potential mediators of tumor invasiveness, is strong in all cells (h). Finally, a generic anti-phospho-tyrosine antibody reveals the general preservation of tyrosine phosphorylation in this tumor section

Because the activation of mitogen-activated protein kinase (MAPK) and members of the Akt pathway can promote glioblastoma cell proliferation, survival, and resistance to radiation, assessment of signaling pathway activation was undertaken in a series of these tumors. Elevated p-MAPK was most strongly associated with poor response to radiotherapy. Elevated p-mTOR, p-p70S6K, and p-MAPK were associated with shorter survival. However, only p-MAPK proved to be an independent prognostic factor after factoring for other clinical variables (Pelloski et al. 2006).

Another study from the same group found that among tumors lacking EGFRvIII expression (a truncated and mutationally activated form of the receptor), the status of phospho-AKT and phospho-ERK was prognostically significant. However, when EGFRvIII was expressed these markers did not provide additional prognostic power (Pelloski et al. 2007). It seems likely that the EGFRvIIInegative tumors represent a more heterogeneous group from the standpoint of molecular pathogenesis and thus benefit more from subclassification using phospho-ERK and phospho-AKT assessment. 
Akt and ERK phosphorylation was investigated by immunohistochemistry in patients with with lymph nodenegative breast cancer. P-Akt, but not p-ERK correlated with HER-2/neu overexpression and was related to reduced tumor apoptosis. No association was found between pAkt or pERK with cell proliferation assessed by Ki67 and mitotic count. P-Akt status proved to be a statistically significant independent prognostic factor (Schmitz et al. 2004). Similarly, a study on head and neck squamous cell carcinomas (HNSCC) found that Akt phosphorylation (Ser 473) correlates with poor prognosis (Massarelli et al. 2005).

Numerous studies have documented the overexpression of the p53 tumor supressor protein in human tumors. P53 function is regulated not only by protein levels, but also by phosphorylation state, which determines stabilization and protection against mdm- 2 . With this in mind, a study investigated phosphorylation at Ser15 and Ser392 in squamous carcinoma and precursor lesions. Although not tested for prognostic value, the findings revealed differential staining patterns with the two PSSAs, suggesting that high levels of Ser392 phosphorylation is an early change in the pathogenesis of SCC (Matsumoto et al. 2004). However, a study on gastroesophageal adenocarcinomas showed no correlation of p53 phosphorylation (Ser15) with p53 mutation, and no correlation with survival. In this setting, analysis of p53 phosphorylation did not appear to have obvious clinical utility. (Puhringer-Oppermann et al. 2006).

A study on prostate cancers examined the phosphorylation Akt and relevant downstream substrates glycogen synthase kinase 3, mTOR, and the forkhead transcription factor-like 1 (FKHRL1). Although most tumors showed elevated phosphorylation of PKB/Akt in the malignant tissue compared with the surrounding benign tissue, only increased phosphorylated-FKHRL1 levels correlated with clinical progression (Jendrossek et al. 2008). Immunohistochemical analysis of renal cell carcinomas pAkt and pS6 was found to be independent prognostic factors (Pantuck et al. 2007).

Because activation of Akt was documented in hormonerefractory prostate cancer (HRPC) and can result in phosphorylation of the androgen receptor (AR), IHC analysis of the phosphorylation status of Akt and AR was tested in a series of prostate cancer tumors. In the hormone-refractory cases, phosphorylated AR (pAR) was associated with worse prognosis (McCall et al. 2008).

Endocrine therapy is an important treatment option for women with estrogen receptor (ER)-positive breast cancer. Information about the activation state of ER could in theory provide more clinically useful information than mere protein expression. A study of ERalpha serine (Ser) 118 and ERalpha Ser167 revealed that low phosphorylation of ERalpha Ser118 and high phosphorylation of ERalpha Ser167 were associated with significantly improved disease-free and overall survival. (Yamashita et al. 2008)This is a good example of another overlooked aspect, namely careful assessment of dephosphorylation of specific epitopes.

Overexpression or activation of the transcription factor c-Jun has been implicated in the pathogenesis of several types of cancer. Phospho-c-Jun staining was correlated with significantly shorter overall survival (Kuo et al. 2006).

PSSAs as predictive markers

A large number of studies have examined the role of immunohistochemical tests forErbB-2 (Her2/Neu) as predictive markers for therapeutic responses of breast cancer to antiHer2 antibodies, culminating in an FDA-approved test (Thor 2001). A logical hypothesis was that assessment of the activation (tyrosine phosphorylation) of Her2 would provide added predictive information. However, immunohistochemistry with the anti-phospho-ErbB-2 antibody had no added benefit to identify those patients most likely to benefit from increased doses of adjuvant chemotherapy (DiGiovanna et al. 2008). However, a similar study but in a different subgroup of breast cancer patients did find clinical utility of PSSAs. The phosphorylation status of Her-2 and EGFR was assessed in Her-2-overexpressing tumor samples from trastuzumab-treated metastatic breast cancers. The presence of ptyr-1248 Her-2 and ptyr-845 or ptyr-1173 EGFR was a strong predictor response of both to anti-Her2 treatment. ptyr-845 EGFR and ptyr-1248 Her-2 were both independent predictors of progression-free survival (Hudelist et al. 2006). The rapid growth of clinical trials of targeted chemotherapeutics will make the development and validation of reliable PSSA IHC testing a high priority for the next decade.

PSSAs as indicators of therapeutic effect

An exciting area application for PSSAs is the assessment of activity in post-treatment tumor samples. As a first step towards clinical application, animal tests were performed using JNJ-10198409, an inhibitor of PDGFR in a nude mouse xenograft model of human colon cancer. The phosphorylation status of phospholipase Cgamma1 (PLCgamma1) was used as a marker of activation of the PDGFR signaling cascade. By using a pair of antibodies in serial sections, one for total (phosphorylated and unphosphorylated forms) and the other for the phosphorylated form of PLCgamma1 (ph-PLCgamma1), an immunohistochemical ratio assay was achieved. Impressively, the authors showed statistically significant, dose-dependent differences in the phospho/total PLCgamma1 ratio among the four treatment groups (vehicle, 25, 50, and $100 \mathrm{mg} / \mathrm{kg}$ 
b.i.d.). This study should serve as a model for future work in animal models as well as in the more complex and heterogeneous human samples (D'Andrea et al. 2005).

A neoadjuvant trial of rapamycin was recently completed in patients with recurrent glioblastoma, whose tumors lacked expression of the tumor suppressor PTEN. Several interesting observations were noted. The magnitude of mTOR inhibition (measured by S6 protein phosphorylation) varied substantially. Also, rapamycin treatment led to Akt activation in seven patients, which was associated with shorter time-to-progression (Cloughesy et al. 2008).

Radiation therapy has profound effects on cell signaling pathways, underlying some aspects of its therapeutic activity. A study of ERK phosphorylation in colorectal cancer revealed that most patients who were phospho-ERK positive before RT converted to ERK negativity after RT (Corn et al. 2008). Although intriguing, further studies are needed to determine if measurement of phospho-ERK can be predictive of response or an indicator of the effect of to radiation therapy.

The use of surrogate cells, obtained noninvasively, to assess chemotherapeutic activity would be a boon for oncologists and much preferred by patients. Buccal mucosa cells were tested as a model in which to assess gefitinib activity in patients with advanced non-small cell lung cancer (Loprevite et al. 2007). Pretreatment and post-treatment cells were tested for expression of p-EGFR, p-ERK and p-AKT. Baseline p-AKT expression, but not that of EGFR, p-EGFR, and p-ERK showed a potentially predictive role, although the study did not reach statistical significance. Further work on buccal cells and other possible surrogate cells will be a fruitful area.

\section{New technologies}

\section{Quantitative image analysis}

Like any immunohistochemical assay, a frequent bottleneck is the unbiased scoring of staining intensity, percent positive cells, and subcellular localizations. Application of an immunofluorescence-based quantitative image analysis system (AQUA) to the study of AKT phosphorylation (Ser473) revealed the marker as a strong negative prognostic factor in oropharyngeal squamous cancer( $\mathrm{Yu}$ et al. 2007). Future applications of this and other image analysis methods using ratio imaging of phospho- to total protein immunoreactivity, as achieved with cultured cells (Mandell and Banker 1996) could provide a readout of relative (\%) phosphorylation, more appropriate for comparisons of multiple samples in which the total amount of the phosphoprotein could vary.

\section{FRET}

Fluorescence resonance energy transfer (FRET) involves the transfer of energy from an excited donor molecule to a nearby $(<7 \mathrm{~nm})$ spectrally overlapping acceptor. Although most widely applied to the study of interacting engineered autofluorescent proteins, FRET can be used to measure the proximity of two appropriately labeled antibodies. Proofof-principle of this method was obtained in cancer cell lines using the acceptor photobleaching fluorescence resonance energy transfer imaging approach to measure the phosphorylation state of EGFR (Keese et al. 2005). In a study on head and neck cancers, a generic anti-phospotyrosine antibody and an anti-EGFR antibody were successfully used to measure EGFR phosphorylation (Kong et al. 2006). FRET efficiency correlated with worsening disease-free survival but not with overall survival. FRET-based methods are much more technically challenging than traditional immunohistochemistry but have some potential advantages. One is the measurement of phosphorylation for proteins for which no good PSSAs are available, by means of a pair of a general (protein-specific) antibody and a generic anti-phosphotyrosine, phosphothreonine, or phosphoserine antibody. The other is the inherent quantitative nature of the measurements. Finally, the method can potentially be used to measure the binding interactions of any pair of molecules in tissue fixed tissue samples.

The hard work ahead: antibody validation and phosphoepitope characterization

An obvious need for PSSAs, as well as for the entire field of immunohistochemistry, is improved antibody validation. A good example of the multiple approaches necessary to truly validate antibodies for quantitative immunohistochemistry was recently published for the case of C-Met (Pozner-Moulis et al. 2007). Much more work will be needed to bring the validation of a large set of PSSAs. Ideally, information gleaned from these types of studies can be made available to the research community via public websites, such as the Abminer site hosted by the National Cancer Institute (http://discover.nci.nih.gov/abminer/).

Because reversible protein phosphorylation is inherently labile, the use of PSSAs requires an additional set of assurances beyond those for general IHC antibodies. For example, surgically excised tissues are often kept at ambient temperatures for long periods in the operating room or in surgical pathology labs during the busy workday. A largely unaddressed issue is the relative stability/lability of various phosphoepitopes ex vivo. We initiated a pilot investigation by growing xenografts in nude mice, excising the tumors, and either fixing immediately, or delaying fixation for four hours (Fig. 3). We were encouraged that some markers, 
Fig. 3 Ex Vivo phosphoepitope lability: preliminary comparison of phospho-NF-kappaB (Ser276) and phospho-CREB (Ser133). To model the potential post-surgical loss of phosphoepitopes, tumor xenografts were grown in nude mice, harvested, and fixed either immediately (a, c), or $4 \mathrm{~h}$ later $(\mathbf{b}, \mathbf{d})$
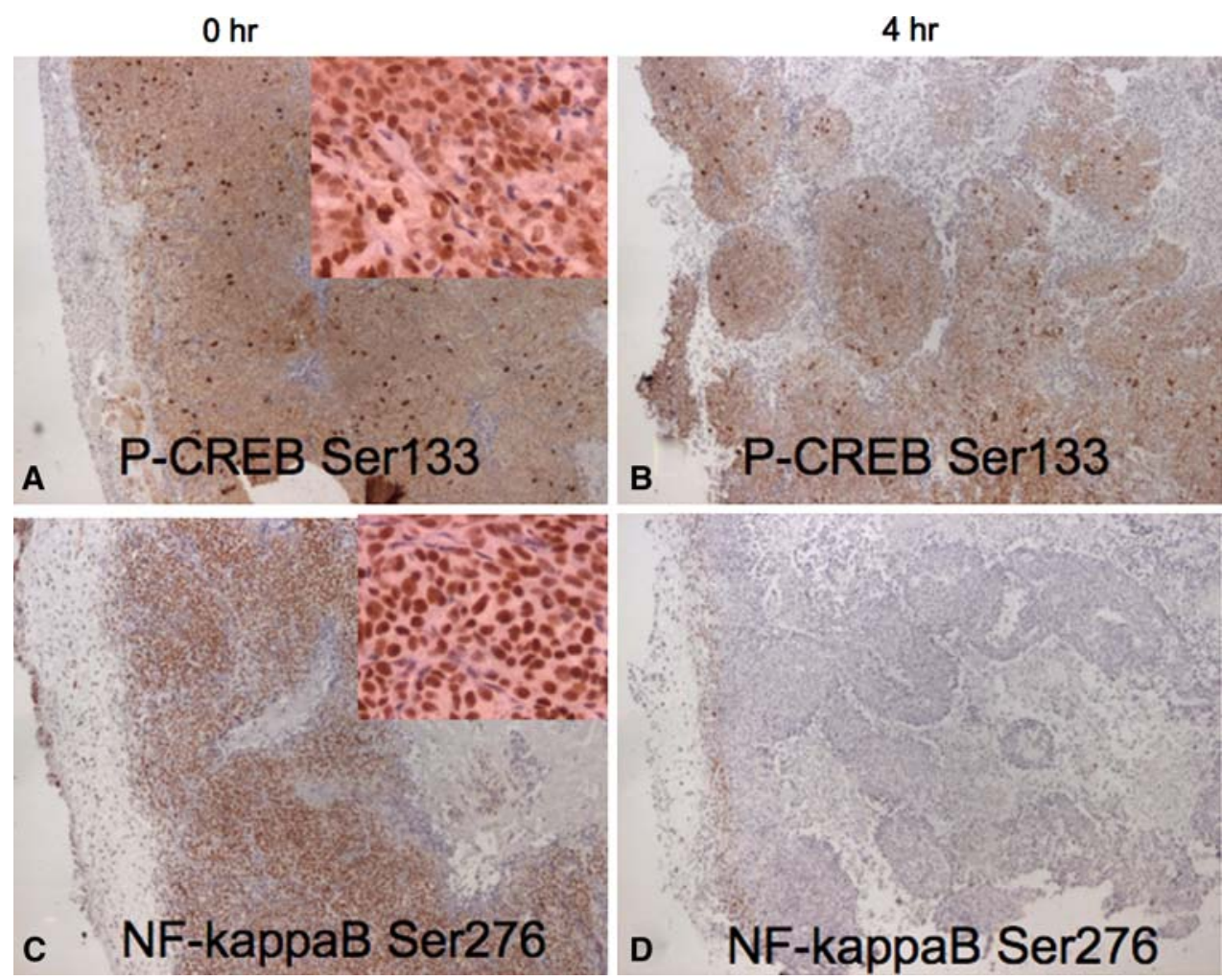

such as phospho-CREB (Ser133) showed no obvious loss of phosphoepitope content at $4 \mathrm{~h}$. However, others, such as phospho-NFkappaB (Ser276) did show apparent loss of immunoreacitivity especially in the interior portions of the tumor. Much more work is needed to quantify phosphoepitope lability using both quantitative western blotting and parallel IHC. If sentinel antibodies sensitive to the general state of cellular phosphorylation levels can be identified, for example, total phosphotyrosine, phosphothreonine, or phosphoserine content, this could provide assurance of reasonable preservation of in vivo phosphorylation states.

\section{Conclusions}

The past 5 years has seen an explosion in the number of immunohistochemical applications of PSSAs, especially in the assessment of human solid tumors. Although it seems logical that knowledge about protein phosphorylation state should provide information above and beyond total protein expression levels, a significant number of studies have shown no added benefit of assessing phosphorylation compared to general antibody IHC. However, enough studies do seem to indicate added prognostic, predictive, and therapeutic monitoring information to warrant further efforts. Without better antibody validations and careful characterization of individual phosphoepitopes in controlled model systems, it will not be possible to properly interpret immunohistochemical results with these potentially powerful reagents.

Acknowledgments I thank George Glass, Peter Cummings, and Tamara Stoops for providing pilot data. I thank Jason Papin, Erwin Gianchandani (Dept. of Biomedical Engineering, U.Va.), David Brautigan and Michael Weber (Dept. of Microbiology) for helpful and provocative discussions.

\section{References}

Cloughesy TF, Yoshimoto K, Nghiemphu P, Brown K, Dang J, Zhu S, Hsueh T, Chen Y, Wang W, Youngkin D, Liau L, Martin N, Becker D, Bergsneider M, Lai A, Green R, Oglesby T, Koleto M, Trent J, Horvath S, Mischel PS, Mellinghoff IK, Sawyers CL (2008) Antitumor activity of rapamycin in a phase I trial for patients with recurrent PTEN-deficient glioblastoma. PLoS Med $5: e 8$

Corn BW, Kovner F, Bek S, Wexler I, Lifschits B, Seger R (2008) ERK signaling in colorectal cancer: a preliminary report on the expression of phosphorylated ERK and the effects of radiation therapy. Am J Clin Oncol 31:255-258

Cortas T, Eisenberg R, Fu P, Kern J, Patrick L, Dowlati A (2007) Activation state EGFR and STAT-3 as prognostic markers in resected non-small cell lung cancer. Lung Cancer 55:349-355

D'Andrea MR, Mei JM, Tuman RW, Galemmo RA, Johnson DL (2005) Validation of in vivo pharmacodynamic activity of a novel PDGF receptor tyrosine kinase inhibitor using immunohistochemistry and quantitative image analysis. Mol Cancer Ther 4:1198-1204

De Camilli P, Carew TJ (2000) Nobel celebrates the neurosciences. Modulatory signaling in the brain. Cell 103:829-833 
DiGiovanna MP, Stern DF, Edgerton S, Broadwater G, Dressler LG, Budman DR, Henderson IC, Norton L, Liu ET, Muss HB, Berry DA, Hayes DF, Thor AD (2008) Influence of activation state of ErbB-2 (HER-2) on response to adjuvant cyclophosphamide, doxorubicin, and fluorouracil for stage II, node-positive breast cancer: study 8541 from the Cancer and Leukemia Group B. J Clin Oncol 26:2364-2372

Fischer EH, Krebs EG (1955) Conversion of phosphorylase b to phosphorylase a in muscle extracts. J Biol Chem 216:121-132

Houben R, Vetter-Kauczok CS, Ortmann S, Rapp UR, Broecker EB, Becker JC (2008) Phospho-ERK staining is a poor indicator of the mutational status of BRAF and NRAS in human melanoma. J Invest Dermatol

Hudelist G, Kostler WJ, Czerwenka K, Kubista E, Attems J, Muller R, Gschwantler-Kaulich D, Manavi M, Huber I, Hoschutzky H, Zielinski CC, Singer CF (2006) Her-2/neu and EGFR tyrosine kinase activation predict the efficacy of trastuzumab-based therapy in patients with metastatic breast cancer. Int $\mathrm{J}$ Cancer 118:1126-1134

Jendrossek V, Henkel M, Hennenlotter J, Vogel U, Ganswindt U, Muller I, Handrick R, Anastasiadis AG, Kuczyk M, Stenzl A, Belka C (2008) Analysis of complex protein kinase B signalling pathways in human prostate cancer samples. BJU Int

Keese M, Magdeburg RJ, Herzog T, Hasenberg T, Offterdinger M, Pepperkok R, Sturm JW, Bastiaens PI (2005) Imaging epidermal growth factor receptor phosphorylation in human colorectal cancer cells and human tissues. J Biol Chem 280:27826-27831

Kong A, Leboucher P, Leek R, Calleja V, Winter S, Harris A, Parker PJ, Larijani B (2006) Prognostic value of an activation state marker for epidermal growth factor receptor in tissue microarrays of head and neck cancer. Cancer Res 66:2834-2843

Kuo RC, Lin CY, Kuo MY (2006) Prognostic role of c-Jun activation in patients with areca quid chewing-related oral squamous cell carcinomas in Taiwan. J Formos Med Assoc 105:229-234

Kuwai T, Nakamura T, Sasaki T, Kim SJ, Fan D, Villares GJ, Zigler M, Wang H, Bar-Eli M, Kerbel RS, Fidler IJ (2008) Phosphorylated epidermal growth factor receptor on tumor-associated endothelial cells is a primary target for therapy with tyrosine kinase inhibitors. Neoplasia 10:489-500

Loprevite M, Tiseo M, Chiaramondia M, Capelletti M, Bozzetti C, Bortesi B, Naldi N, Nizzoli R, Dadati P, Kunkl A, Zennaro D, Lagrasta C, Campanini N, Spiritelli E, Camisa R, Grossi F, Rindi G, Franciosi V, Ardizzoni A (2007) Buccal mucosa cells as in vivo model to evaluate gefitinib activity in patients with advanced non small cell lung cancer. Clin Cancer Res 13:6518-6526

Mandell JW (2003) Phosphorylation state-specific antibodies: applications in investigative and diagnostic pathology. Am J Pathol 163:1687-1698

Mandell JW, Banker GA (1996) A spatial gradient of tau protein phosphorylation in nascent axons. J Neurosci 16:5727-5740

Manning G, Whyte DB, Martinez R, Hunter T, Sudarsanam S (2002) The protein kinase complement of the human genome. Science 298:1912-1934

Massarelli E, Liu DD, Lee JJ, El-Naggar AK, Lo Muzio L, Staibano S, De Placido S, Myers JN, Papadimitrakopoulou VA (2005) Akt activation correlates with adverse outcome in tongue cancer. Cancer 104:2430-2436
Matsumoto M, Furihata M, Kurabayashi A, Ohtsuki Y (2004) Phosphorylation state of tumor-suppressor gene $\mathrm{p} 53$ product overexpressed in skin tumors. Oncol Rep 12:1039-1043

McCall P, Gemmell LK, Mukherjee R, Bartlett JM, Edwards J (2008) Phosphorylation of the androgen receptor is associated with reduced survival in hormone-refractory prostate cancer patients. Br J Cancer 98:1094-1101

Nairn AC, Detre JA, Casnellie JE, Greengard P (1982) Serum antibodies that distinguish between the phospho- and dephospho-forms of a phosphoprotein. Nature 299:734-736

Nieto Y, Nawaz F, Jones RB, Shpall EJ, Nawaz S (2007) Prognostic significance of overexpression and phosphorylation of epidermal growth factor receptor (EGFR) and the presence of truncated EGFRvIII in locoregionally advanced breast cancer. J Clin Oncol 25:4405-4413

Pantuck AJ, Seligson DB, Klatte T, Yu H, Leppert JT, Moore L, O’Toole T, Gibbons J, Belldegrun AS, Figlin RA (2007) Prognostic relevance of the mTOR pathway in renal cell carcinoma: implications for molecular patient selection for targeted therapy. Cancer 109:2257-2267

Pelloski CE, Lin E, Zhang L, Yung WK, Colman H, Liu JL, Woo SY, Heimberger AB, Suki D, Prados M, Chang S, Barker FG 3rd, Fuller GN, Aldape KD (2006) Prognostic associations of activated mitogen-activated protein kinase and Akt pathways in glioblastoma. Clin Cancer Res 12:3935-3941

Pelloski CE, Ballman KV, Furth AF, Zhang L, Lin E, Sulman EP, Bhat K, McDonald JM, Yung WK, Colman H, Woo SY, Heimberger AB, Suki D, Prados MD, Chang SM, Barker FG 2nd, Buckner JC, James CD, Aldape K (2007) Epidermal growth factor receptor variant III status defines clinically distinct subtypes of glioblastoma. J Clin Oncol 25:2288-2294

Pozner-Moulis S, Cregger M, Camp RL, Rimm DL (2007) Antibody validation by quantitative analysis of protein expression using expression of Met in breast cancer as a model. Lab Invest $87: 251-260$

Puhringer-Oppermann F, Stahl M, Keller G, Sarbia M (2006) Lack of prognostic impact of $\mathrm{p} 53$ gene mutation and $\mathrm{p} 53$ phosphorylation at serine 15 in multimodally treated adenocarcinomas of the gastroesophageal junction. J Cancer Res Clin Oncol 132:433-438

Roskelley CD, Bissell MJ (2002) The dominance of the microenvironment in breast and ovarian cancer. Semin Cancer Biol 12:97-104

Schmitz KJ, Otterbach F, Callies R, Levkau B, Holscher M, Hoffmann O, Grabellus F, Kimmig R, Schmid KW, Baba HA (2004) Prognostic relevance of activated Akt kinase in node-negative breast cancer: a clinicopathological study of 99 cases. Mod Pathol $17: 15-21$

Thor A (2001) HER2-a discussion of testing approaches in the USA. Ann Oncol 12(Suppl. 1):S101-S107

Yamashita H, Nishio M, Toyama T, Sugiura H, Kondo N, Kobayashi S, Fujii Y, Iwase H (2008) Low phosphorylation of ER \{alpha\} serine 118 and high phosphorylation of ER \{alpha\} serine 167 improve survival in ER-positive breast cancer. Endocr Relat Cancer

Yu Z, Weinberger PM, Sasaki C, Egleston BL, Speier WFT, Haffty B, Kowalski D, Camp R, Rimm D, Vairaktaris E, Burtness B, Psyrri A (2007) Phosphorylation of Akt (Ser473) predicts poor clinical outcome in oropharyngeal squamous cell cancer. Cancer Epidemiol Biomarkers Prev 16:553-558 